\title{
An adolescent with newly diagnosed diabetes mellitus presenting with edema: Questions
}

\author{
Ashna Pudupakkam $^{1} \cdot$ Faris Hashim $^{1}$ (D) $\cdot$ Matthew Stephen ${ }^{1} \cdot$ Stephanie Blasick $^{1}$ \\ Received: 11 December 2021 / Revised: 10 January 2022 / Accepted: 11 January 2022 / Published online: 3 February 2022 \\ This is a U.S. government work and not under copyright protection in the U.S.; foreign copyright protection may apply 2022
}

Keywords Insulin edema - Diabetes mellitus · Weight gain

\section{Case summary}

A 16-year-old girl with newly diagnosed type 1 diabetes (T1D) presented to the emergency department with edema associated with weight gain. Four days prior, she was diagnosed with new-onset diabetes after presenting to the hospital with glucose $550 \mathrm{mg} / \mathrm{dL}$, serum ketones $6.1 \mathrm{mmol} / \mathrm{L}$, and development of symptoms over the preceding 3 months. Hemoglobin A1c was elevated at 16.1, and GAD-65 and zinc transporter 8 antibodies were positive. She was started on multiple daily injection insulin therapy with improvement in glucose and symptoms. Following discharge, she developed edema of bilateral legs which spread to involve her feet, arms, and face. She also reported a weight gain of $8 \mathrm{~kg}$ (41st percentile, $z$-score increased from -0.2 to 0.5 ) since discharge. She denied associated fever, shortness of breath, chest pain, abdominal pain, nausea or vomiting, and changes in urination.

At ED presentation, vital signs were within normal limits for age. Physical exam was significant for pitting edema of the lower extremities. She did not have pulmonary crackles or hepatomegaly. Initial labs were significant for hypoalbuminemia (serum albumin $2.7 \mathrm{mg} / \mathrm{dL}$ ) and hyperglycemia (serum glucose $191 \mathrm{mg} / \mathrm{dL}$ ). Complete blood count and comprehensive metabolic panel were otherwise within normal limits. Urinalysis showed glucosuria $(3+$ glucose $)$ without proteinuria. Microalbumin to creatinine ratio was within normal limits. Abdominal ultrasound showed increased echogenicity around the medullary pyramids of bilateral kidneys.

\section{Questions}

1. What is the most likely diagnosis?

2. What are alternative diagnoses to consider?

3. What is the treatment of this condition?

Publisher's note Springer Nature remains neutral with regard to jurisdictional claims in published maps and institutional affiliations.
The answers to these questions can be found at https://doi.org/10. 1007/s00467-022-05463-y

Faris Hashim

faris.hashim@bswhealth.org

1 Baylor Scott \& White Health, Temple, TX, USA 